\title{
Understanding Experimental Economics ${ }^{1}$
}

Carsten S. Nielsen

Post.doc, Department of Psychology, University of Copenhagen

Alexander Sebald

Associate Professor, Department of Economics, University of Copenhagen

Edward J.D. Webb

Ph.D. Fellow, Department of Economics, University of Copenhagen

Experiments have become a well-established methodological tool in economics. The development of experimental economics and the diversification of experimental methods have equipped economists with new and powerful means of scientific investigation. Their worth is readily demonstrated in the exciting and promising results they have produced, and will continue to produce. Against the background of this success story, our selective discussion critically highlights four important aspects of experimentation in economics. We concentrate on the role and importance of material incentives, potentially confounding experimenter demand effects and strategies to minimise these, the no deception rule as well as the issue of external validity.

\section{Introduction}

An observer of a typical economic experiment would see the following: a group of subjects, most likely undergraduates majoring in economics, arrive at a laboratory having been recruited online. The laboratory consists of a number of computers surrounded by screens to give privacy to the users. While the subjects sit at these computers, the experimenter reads out instructions from a sheet and the subjects perform the tasks they have been set, with careful monitoring to check that no communication occurs. Afterwards the experimenter pays each subject in private according to the anonymous decisions made in the experiment.

That is what an observer would see, but what exactly is an economic experiment? Taking the latter half of the name first, it is an experiment: a controlled data genera- ting process. One factor of interest (the 'treatment variable') is varied at a time, while all other factors which influence behaviour are held constant. This controlled variation of factors is crucial to be able to draw causal inference.

Secondly, it is economic. The majority of economic experiments are either theory-testing or theory-suggesting. Economic theory provides a framework and tools for describing and analysing economic situations. It makes assumptions about individuals' behaviour and the institutions in which they interact to derive predictions and to provide explanations for economic phenomena. More specifically, an economic theory is a description of an economic environment, specifying the abstract agents, the actions they can take, their information, and how they evaluate each possible outcome. When the economic environment is in place, predictions about outcomes are derived by applying 'solution concepts' that describe how assumptions about the economic environment translate into observed outcomes. This is an extremely powerful framework that provides intellectual clarity, mathematical rigour and tractable formalisations.

Economic experiments form a dialogue with economic theory: theory is adapted for testing in the laboratory, persistent anomalies or violations are identified, the theory is modified or replaced, and the process of refinement begins anew. An experiment, like the theory it tests, is an abstraction from the real world. Experiments are, by definition, descriptively inaccurate. However, this does not mean that they are useless. In particular, experiments make the test of logical implications of behavioural assumptions explicit and concrete. An experiment can thus be seen as a boundary case, but understanding boundary cases is critical for scientific development. This does, however, not relieve economists from developing 
new theories based on more descriptively accurate data, but it provides an important backdrop against which new theory-suggesting experiments can be conducted. As Mäki (2005) succinctly puts it: experiments are models and models are experiments.

What would subjects in an economic experiment then observe? When sitting in front of their computers, they make decisions involving real material consequences. Subjects are told the rules of the experiment, that is, the actions that they are allowed to take within the experimental environment and how these actions translate (often probabilistically) into some outcome medium (usually money). Notice that within this scheme, one needs to say nothing at all about the hypothesis being tested. Having explained the rules, all the experimenter needs to say is something along the following lines: „here is the situation in which you, the subject, has to make a decision, these are the possible implications of your decisions do what you will".

Many of the ways in which the experimental economic methodology differs from other experimental fields, especially some areas of experimental psychology, stem from the objective of internal validity. In this selective review, we will discuss some of the dimensions that are most characteristic for economic experiments and compare them (where suitable) to methodologies in other fields of science. Economic theories predict how economic agents will act in the presence of real material consequences. Therefore, performance contingent payoffs are critical for economics experiments (Section 2). Theories assume that individuals only respond to the incentives found in their economic environment, rather than a motivation to please the experimenter. Thus the experimenter should be careful not to lead subjects by the hand and thereby avoid experimenter demand effects (Section 3). Theories also assume that agents understand and believe the stated relationship between their actions and their payoffs, thus deception would endanger this belief and is almost nonexistent (Section 4). We then debate the external validity of experiments and the advantages and disadvantages of field experiments with regards to this issue (Section 5). Finally, we conclude (Section 6).

\section{Material Consequences}

Experiments in economics involve material consequences of the actions that subjects take (most often monetary consequences). For economists, hypothetical questions are not considered very informative. Economic theory makes no predictions regarding what economic agents say that they would do in hypothetical situations, only what they actually do when faced with a decision with real consequences. It is thus critical for theory testing in economics that subjects actually face the consequences assumed by the theory. This has led to the practice of performance contingent payoffs in experimental economics, where subjects' compensation is contingent on the actions they take in a way that is consistent with the theory being tested. These payoffs replace the flat-fee payments that are often used in other experimental fields. There is overwhelming consensus among economists that material consequences in the form of money affect performance for the better (Davis and Holt 1993, Roth 1995, Smith 1998). Theoretically, there are at least two reasons for this. First, everyone values money positively, in contrast with, for example, consumer goods that may be valued differently among subjects. Second, money is non-satiable -more is always better. That said, some experiments use non-monetary payoffs. For example, in some decisionmaking experiments, subjects are asked to value different goods which they receive after the experiment has ended. Such valuations are typically implemented by using the Becker-DeGroot-Marschak mechanism (Becker et al. 1964), which truthfully elicits subjects' willingness-topay for these goods in monetary terms.

Few experiments published in economics provide no payoffs at all. One important exception, with great influence on the economic literature, is rooted in psychology - namely the experimental documentation of systematic departures from the predictions of rational decisionmaking (Tversky and Kahneman 1974, Kahneman and Tversky 1979, Tversky and Kahneman 1981). Their documentation is based on hypothetical decision-making questions such as:

\section{Choose between:}

$\begin{array}{lll}\text { A. } & \text { 2,500 with probability } & .33 \\ \text { B. } & \text { 2 with probability } & .67 \\ & \text { 2,400 with probability } & .34 \\ & \text { with probability } & .66\end{array}$

Most economists think that such questions, and the associated answers, are less useful. We typically think of 'cognitive effort' as a scarce resource that subjects have to allocate strategically. If subjects are not paid contingent on their actions, so we argue, they will not invest cognitive effort to avoid decision errors. However, if some payoff is provided, the subjects' decisions will be closer to what is predicted by theory and result in a reduction in the variance of decision error (Smith and Walker 1993). The severity of not paying subjects is not obvious. One could, for example, argue that subjects answering the aforementioned hypothetical question use their intuition, and have no particular cognitive cost associated with making the 
decision (Thaler 1987). When, for example, asked for the time of day, few will not truthfully reveal it.

When paying subjects in experiments, an issue is often how much they should be paid. The norm in experimental economics is that subjects must be paid for their time. Thus, the average monetary payoff (or non-monetary equivalent) should be translated into an hourly wage roughly equal to the salaries of student jobs. Since performance contingent payoffs may become expensive, there are a number of 'tricks' that economists can use in order to stretch limited funds. First, if subjects face exactly the same situation repeatedly, we sometimes choose one round at random for real payment. Since subjects are told in advance that one round will be randomly selected but not which one, it is still in their interest to exert the same effort in each round. Second, for experiments with many subjects, we sometimes select one subject at random for real payment. Again, subjects are told in advanced that one will be chosen at random. While both these techniques reduce expenditure, experimental economists using these designs typically make the expected earnings of any given subject equal to the wage rate.

There is, however, no guarantee that monetary consequences will suitably incentivise subjects in all experiments and that subjects' decisions will move closer to theory. For example, Camerer and Hogarth (1999) found that monetary consequences mattered more in decisionmaking experiments than in games and market experiments. One reason for this finding could be that in tedious decision-making experiments, subjects require extrinsic monetary payoffs to exert effort, whereas in for example, games, they are intrinsically motivated to compete. Having said that, the practice of paying subjects in a performance contingent manner is in economics considered critical to the internal validity of the experiment and the objective of testing the theory in question. Internal validity requires the maximum possible level of control over all relevant dimensions of the experiment. The less control there is, the less the experimenter can claim that the only source of any observed treatment difference is the treatment variation they have exposed different experimental subjects to. As was mentioned earlier, virtually no experiments without material consequences are now published in economics.

\section{Experimenter Demand Effects}

Experimenter demand effects, which are changes in the behaviour of experimental subjects caused by their belief about what the experimenter expects of them, can also weaken internal validity. A classic and very explicit example of demand effects is Milgram's (1963) experiment on obedience. Here experimental subjects had to deliver electric shocks (the treatment variable) to others, under the pressure of the experimenter, not knowing that these shocks were in fact fictitious.

Although experimental economists often worry about experimental demand effects, not all experimenter demand effects are actually harmful to the conclusions that can be drawn. In fact, sometimes they might actually be helpful to achieve other goals, or they are the very object under investigation and thus necessary to the experimental set-up. To illustrate the first point, Andersen et al. (2011) conducted an ultimatum experiment in a poor region of India. One aim was to investigate people's reactions to very low proposals in the ultimatum game. This is difficult because low proposals are usually not made by proposers in ultimatum games making it impossible to observe responders' reactions to such low offers. To overcome this problem, they induced low offers by adding a specific message to the instructions:

"Notice that if the responder's goal is to earn as much money as possible from the experiment, he/she should accept any offer that gives him/her positive earnings, no matter how low. This is because the alternative is to reject, in which case he/she will not receive any earnings. If the responder is expected to behave in this way and accept any positive offer, a proposer should offer the minimum possible amount to the responder in order to leave the experiment with as much money as possible. That is, if the responder that you are matched with aims to earn as much money as possible, he/she should accept any offer that is greater than zero. Given this, making the offer that gives the lowest possible earnings to the responder will allow you to leave the experiment with as much money possible." (Andersen et al. 2011, 3430)

Clearly this instruction informs proposers that the rational decision is to offer the lowest possible amount, creating an experimenter demand effect. Secondly, the aforecited article by Milgram was studying the role of obedience making the experimenter demand effect the object of study. These two studies together show that experimenter demand effects may be desirable to achieve the experimenter's objective. However, in all situations in which experimenter demand effects impact and confound the results that one sets out to study, they are detrimental as they bring into question the conclusions that experimenters can draw. 
In experiments, subjects are placed into micro systems (Smith 1982, 1994) by the experimenter putting him into a vertical authority relationship with the subjects participating. The experimenter is the creator of this micro system, who has not only a better understanding of all its elements but also the better understanding of the background of the study. This vertical authority relationship is one of the roots of experimenter demand effects. Hence, factors that influence the vertical authority relationship in the experiment are likely to influence confounding experimenter demand effects.

As Zizzo (2010) and others have already pointed out, crucial factors that determine and influence this relationship in experiments are (i) the very detailed and explicit instructions that subjects are given at the start of the experiment, (ii) how possible actions are translated into rewards, and (iii) the laboratory setting, for example, the fact that subjects are not allowed to talk, that they have to sit at their desks while the experimenter and assistants walk around to check on the progress of the experiment and answer questions. It is here that possible improvements or solutions to the problem of confounding experimenter demand effects lies.

Instructions given to subjects in economic experiments are usually very detailed and written in neutral language. They are elaborate as it is very important for experimenters to make sure that subjects fully understand the economic environment they are confronted with and to allow for meaningful replications of the experimental study in other settings at other times. Subjects in economic experiments should not make any decisions without understanding the possible consequences of their actions. Furthermore, neutral language is used to abstract from any specific and narrow real world context and thus increases the generalisability of the results.

For example, consider the following excerpt from the hypothetical instructions given to a subject in a two player 'principal-agent experiment' in which subjects allocated to the role of the agent (Person B) have to work for a matched principal (Person A), and the principal pays the agent a wage out of the generated profit:

You are Person B and you are paired with another person: Person A.

\section{Explanations to Part 1:}

Part 1 consists of two stages called 'Task' and 'Guessing/ Feedback'.

Task: In the first stage you have to choose how much effort to spend on a task. The task consists of clicking boxes...
Clearly, this way of presenting the agent's task (that is, „you have to choose...") entails the risk of an experimenter demand effect. The instructions are detailed and clear (point (i) above), however, Person A might believe that the experimenter expects him to click boxes away. However, if agents perceive it as their obligation to work in this context, it might seriously hamper an analysis of, for example, the impact of different incentive systems on the agents' performance.

To assess the potential improvement of an alternative way of presenting the agent's task, consider now the following excerpt:

You are Person B and you are paired with another person: Person A.

\section{Explanations to Part 1:}

Part 1 consists of two stages called 'Task' and 'Guessing/ Feedback'.

Task: In the first stage you can decide how much effort to spend on a task that consists of clicking boxes...

Rather than saying that the agent "has to do" something, the rewritten instructions now say „you can choose how much effort to spend..." presenting the task in a much less normative way. As suggested before, limiting the normative content of instructions minimises the risk of undesired experimenter demand effects in economic experiments.

Furthermore, in the context of this example, one can also influence the agent's sense of obligation by offering an alternative action (point (ii) above). For example, the agent could be offered to always be able to switch to a video for the remaining duration of the clicking task. Such an option changes the incentives the agent is confronted with which in turn might decrease the agent's feeling of obligation with regard to the effort task or prevent them from doing it out of sheer boredom. Such a strategy has been used in Caria and Falco (2014) who equipped the experimental rooms with televisions so as to minimise experimenter demand effects in the setting of a real effort task.

Finally, regarding the atmospheric issues (point (iii) above), the experiment could be moved out of the laboratory to the field or on the Internet so that experimental subjects do not actually have to come to the laboratory to participate. Field and Internet experiments might help to break up or weaken the vertical authority relationship most apparent in a laboratory environment. We discuss field experiments further in Section 5. 


\section{No Deception}

When striving for internal validity in experimental economics, the use of deception is also ill-advised (see Hey 1991, Davis and Holt 1993, Friedman and Sunder 1994). The basis for this advice is the assertion that second-guessing the intent of the experiment jeopardises control and internal validity. It is in economics assumed that the economic agents described by theory understand the rules under which they operate. If subjects in an experiment designed to test economic theory are deceived about any of these rules, then the validity of their actions can be called into question. The experimenter potentially looses control and he no longer knows the nature of the theory being tested by his experiment.

A second reason for advising against deception in economics has to do with the public good of the subject's trust in the experimenter. The concern here is that many experimental laboratories use an overlapping pool of subjects. Thus, a public good problem exists in which experiencing deception in one experiment may cause subjects to second-guess the rules of other experiments. Clearly, maintaining this public good involves a difficult trade-off where private benefits to the experimenter can end up tainting the subject pool. The fact that deception is seldom used in economics and is received with hostility by referees and editors makes the non-deceptive status quo maintainable in experimental economics.

In spite of the prominence of the no deception norm in experimental economics, there exist few guidelines on what constitutes deception. We believe that there is good reason to distinguish between various concepts that one might be tempted to call deception. For instance, a distinction is made between deception by commission - telling the subjects things that are not true - and deception by omission - not telling them everything. In experiments with deception by omission, subjects are typically not aware of every contingency of the experiment. However, if they ask about contingencies we do not lie: we do not deceive them by commission. Deception by omission has, for example, in experimental economics been used to surprise subjects with additional actions when they thought the experiment had ended (Andreoni 1988, Croson 1996; Boles et al. 2000, Croson et al. 2003).

We want to stress that the use of deception by omission comes with at least one caveat. When testing the assumptions of a theory or conducting theory-suggesting experiments, it is a crucial feature that everything is well specified - particularly the rules under which the economic agent in the theory is operating. The experimenter has to tell the subject everything that is assumed to be known by the agent of that theory. Otherwise, the experimental environment is no longer an appropriate representation of the theory in question. This does not, however, mean that the rules need to be common knowledge in the experiment. If the theory being investigated prescribes that agents have different or imperfect knowledge about the rules, then the experimenter should not tell all subjects all the rules. If we, for example, want to experimentally test the interactive unawareness theory of Heifetz et al. (2006, 2008, 2013a, 2013b), in which an economic agent may conceive only some aspects of the environment he acts in and remain unaware of others, then we should not tell the subject (acting the role of the agent) about things in the experiment he is unaware of in the tested theory.

Few examples of deception by commission - telling subjects things that are not true - can be found in the economics literature. One rare economic study that employs deception by commission is Schneider and Pommerehne (1983), who experimentally examine the free-rider problem in public goods provision. In order to control for the tendency that free riding increases with group size (at a low cost), they deceive subjects by having them think that there exist participating subjects at two other universities. Furthermore, the actual subjects were wrongly told that the actions made at the other universities were already known, so that the experimenter could determine the outcome of the experiment immediately after the real bidding. The problem is, of course, that if such deception by commission is detected, subjects will start to second-guess the intent of the experiment. The experimenter could try to avoid the possibility of being detected by making sure that subjects did not make contact after the experiment or by performing the experiment with subjects visually separated from each other. The problem with this is that such procedures can make subjects suspicious of deception in the experiment. Worst-case scenario, experimental economists would be saddled with a reputation for routinely deceiving subjects, rendering proper experimental control extremely difficult.

We believe that deception by omission of subjects in an experiment can be used when theory prescribes heterogeneous knowledge among agents, as was the case with the interactive unawareness theory. However, one should refrain from using deception by commission in experimental economics, although we do acknowledge that there have been psychological experiments that used deception by commission to spectacularly good effect. For example, the aforecited Milgram experiment would lose its force if some subjects had not believed that they were administering dangerous shocks to an unwilling subject. If, however, subjects in Milgram's experiment had reasons to doubt the experimenter, then all the advantage of deception is lost. To mitigate the undesirable effects of 
deception, experimental psychologists often debrief their subjects, revealing the deception and asking them not to tell other potential subjects about their knowledge. Although this is recommended and often required by ethical committees, to economists this practice only enhances the likelihood of recruiting subjects who enter the experiment expecting deception and further weakens internal validity.

Further discussions on whether or not deception can be used in experiments, and especially the methodological differences between experimental psychology and economics, can be found in Bonetti (1998), Hey (1998), McDaniel and Starmer (1998), Hertwig and Ortmann (2001), Ortmann (2002) and Jamison et al. (2008).

\section{External Validity}

We have until now argued that experiments are data generating processes that should be used for theory-testing and theory-suggesting enquiries, and that the experimenter should do his utmost to ensure experimental control and internal validity in an attempt to ensure that it is an appropriate test of some theory.

However, an important question is whether behaviour inside the laboratory is a good indicator of behaviour outside the laboratory - whether it is externally valid. Recent work by Levitt and List (2007a, 2007b, 2008) has questioned what we can learn from laboratory experiments. They argue that the implicit assumption underlying the interpretation of data in many laboratory experiments, that the insights gained in the experiment can easily be extrapolated to the real world, is invalid.

For example, one common objection is that the stakes (the material consequences) in laboratory experiments are trivial. The effects of high stakes in laboratory experiments are, however, mixed and seem to be context dependent (Camerer and Hogarth 1999). Reciprocity, for instance, does not disappear in the gift exchange experiment when subjects are paid an equivalent of three months' income (Fehr et al. 2013). But just how often do people make decisions involving monthly incomes, and how representative would such high stake experiments be of the many decisions people make on a daily basis involving small stakes? Another often heard objection to laboratory experiments is that the number of subjects is often too small, which seriously endangers the external validity of the results. However, we believe that this objection draws attention away from the more important issues: namely the use of proper econometric methods to analyse the data. Efficient econometric methods exist for analysing small sample experiments (Siegel and Castellan 1988, Manski 2005, Heckman et al. 2013). Finally, it has been argued that the external validity of labora- tory experiments is limited because subjects in laboratory experiments behave differently to how they would behave while not being under scrutiny. However, being scrutinised is not an exclusive feature of the laboratory: many decisions in the outside world are observed. In the laboratory we can, however, systematically study the relevance of scrutiny by varying the degree of anonymity, for example, investigating differences between subjectsubject anonymity and full anonymity between subjects and the experimenter.

Moreover, as theorists we seek a general understanding of the way in which agents' characteristics, incentives, rules, information and payoff structures influence economic behaviour. We do not require that student subjects in a laboratory setting designed to resemble a real world environment behave in the same way as professionals. Behaviour may differ because the subject population is different or because of many differences between the laboratory and the outside world. The maintained assumption in experimental economics, as we see it, simply asserts that if those differences could be held constant or controlled for econometrically, behaviour in the laboratory and in the wild would be the same. This is the core idea of what Smith (1982) termed 'parallelism'. The chemist could make his sterile test tubes more like the real world by adding dirt. The fact that he chooses not to does not render chemistry experiments irrelevant. Or expressed more eloquently:
"The issue of realism, however, is not a distinctive feature of laboratory versus field data. The real issue is determining the best way to isolate the causal effect of interest... The casual reader may mistakenly interpret arguments about realism as an effective critique against the laboratory, potentially discouraging laboratory experimentation and slowing down the production of knowledge in economic and other social sciences." (Falk and Heckman 2009, 536)

If the goal is to understand the general principles of behaviour, then both experimental and naturally occurring data must contribute in parallel to theory. Whether or not laboratory findings are externally valid is not crucial. Furthermore, if there exist special features of a laboratory experiment that might limit generalisability, this can be relaxed, if necessary, to better mirror a particular real world environment. Just as scientific theories are simplified representations of complex real world systems, so are philosophies of science simplified representations of complex, varied and overlapping methodologies and practices. 
To bring to a close the philosophical discussion, it should be admitted that external validity is relatively more important from an economic practitioner's perspective. Especially, since laboratory experiments have turned out to be valuable in solving practical problems that arise in, for example, implementing matching markets (Kagel and Roth 2000) or spectrum auctions (Holt and Goeree 2010).

One practical response to questions of external validity in recent years has been to move outside the laboratory into the field. Harrison and List (2004) define field experiments as being methodologically complementary to traditional laboratory experiments. Field experiments generate data from a particular economic environment where subjects themselves choose the environment. The usual cited advantage of a field experiment is that it more closely matches the target domain of theory and hence has greater external validity. A caveat to this assertion, though, is that a field experiment is close to one specific target domain. There is no a priori reason to suppose that target domain A more closely resembles target B than does laboratory L. Another drawback of field experiments is that what they gain in external validity, they lose in internal validity (Guala 2005, Schram 2005). Harrison (2005) raises an objection arguing that control is important in both the laboratory and in the field, and that a field setting is not an excuse for poor experimental control. This is indisputable. Nevertheless, from a practical point of view, control is harder in the field than in the laboratory which is an environment specifically created for the optimal control of economic experiments.

The rise and spread of field experiments might be thought to herald the demise of the traditional laboratory experiment. We do not think that this is the case. Neither should it be assumed that there is a hierarchy of economic experimental methods, such as it is found with the different phases of clinical trials. Laboratory and field experiments are different tools, each of which is available to the economist to approach different problems in different ways. One can combine laboratory and field experiments to better understand the mechanisms observed in the field. For example, this can be done by eliciting preferences and relating these preferences to observed behaviour in the field (Karlan 2005, Todd and Wolpin 2006).

Finally, an essential prerequisite for external validity is that experimental findings do not disappear upon replication. As in other social sciences, experimental economics has put less emphasis on replication and more on 'eye-catching' novel results. Unfortunately, such an emphasis can potentially threaten the external validity of experimental results. As shown by Maniadis et al. (2014), a narrow focus on having statistical significance (a p- value of less than 0.05 ) can lead to the publication of a large number of false positive results. As a consequence, many significantly novel results could disappear upon replication. One solution is to also take the statistical power of experimental findings into account. Another solution, proposed by psychologist and Nobel laureate in economics Daniel Kahneman ${ }^{2}$, is to have several research groups take part in a 'daisy chain' of replication with each research group undertaking to replicate the results of another research group.

We believe that a few independent replications can dramatically increase the chances that the original finding is true. As Fisher (1935) emphasised, a cornerstone of the experimental science is replication.

\section{Conclusion}

We conclude by restating our main point. The development of experimental economics and the diversification of experimental methods have equipped economists with new and powerful means of scientific investigation. Their worth is readily demonstrated in the exciting and promising results they have produced and continue to produce. In recent years, economists have hotly debated which form of experimentations is most informative. This discussion has been fruitful and will in no doubt continue in the years to come. In this context it is important to acknowledge that theoretical models, naturally occurring data, field data and laboratory data are complements, not substitutes. Each can improve economic understanding. None of these are to be preferred -there does not exit a valid hierarchy among these methods and the issue of generalisability is universal to them all.

\section{Bibliography}

Andersen, S, S Ertac, U Gneezy, M Hoffman \& JA List 2011, 'Stakes Matter in Ultimatum Games', American Economic Review, vol. 101, no. 7, pp. 3427-3439.

Andreoni, J. 1988, 'Why free ride? Strategies and learning in public goods experiments', Journal of Public Economics, vol. 37, no. 3, pp. 291-304.

Boles, T, R Croson \& J Murnighan 2000, 'Deception and Retribution in Repeated Ultimatum Bargaining', Organizational Behavior and Human Decision Processes, vol. 83, no. 2, pp. 235-259.

Bonetti, S 1998, 'Experimental economics and deception', Journal of Economic Psychology, vol. 9, no. 3, pp. 377-395.

Caria, SA \& P Falco 2014, 'An experimental study of trust in the labour market. Do employers trust workers too little', CSAE Working Paper WPS / 2014-07. Croson, R, T Boles \& JK Murnighan 2003, 'Cheap talk in bargaining experiments: lying and threats in ultimatum games', Journal of Economic Behavior \& Organization, vol. 51, no. 2, pp. 143- 159 . 
Croson, RTA 1996, 'Information in ultimatum games: An experimental study', Journal of Economic Behavior \& Organization, vol. 30, no. 2, pp. 197-212.

Davis, D \& C Holt 1993, Experimental Economics, 1. edn., Princeton University Press, Princeton.

Fisher, RA 1935, The Design of Experiments, Oliver and Boyd, Edinburgh.

Friedman, D \& S Sunder 1994, Experimental Methods: A Primer for Economists, 1. edn., Cambridge University Press, Cambridge.

Guala, F 2005, The Methodology of Experimental Economics, 1. edn., Cambridge University press, Cambridge.

Harrison, GW 2005, 'Field Experiments and Control', in J Carpenter, GW Harrison \& J List (red.), Field Experiments in Economics. Research in Experimental Economics, Elsevier, Oxford.

Harrison, GW \& JA List 2004, 'Field Experiments', Journal of Economic Literature, vol. 42, no. 4, pp. 1009-1055.

Heifetz, A, M Meier \& BC Schipper 2008, 'A Canonical Model for Interactive Unawareness', Games and Economic Behavior, vol. 62, no. 1 , pp. 304-324.

Heifetz, A, M Meier \& BC Schipper 2013a, 'Dynamic Unawareness and Rationalizable Behavior', Games and Economic Behavior, vol. 81, pp. 50-68.

Heifetz, A, M Meier \& BC Schipper 2006, 'Interactive Unawareness', Journal of Economic Theory, vol. 130, no. 1, pp. 78-94.

Heifetz, A, M Meier \& BC Schipper 2013b, 'Unawareness, Beliefs, and Speculative Trade', Games and Economic Behavior, vol. 77, no. 1, pp. 100-121.

Hertwig, R \& A Ortmann 2001, 'Experimental practices in economics: A methodological challenge for psychologists?', The Behavioral and Brain Sciences, vol. 24, no. 3, pp. 383-451.

Hey, JD 1998, 'Experimental economics and deception: A comment', Journal of Economic Psychology, vol. 19, no. 3, pp. 397-401.
Hey, JD 1991, Experiments in Economics, 1. edn., Basil Blackwell, Oxford.

Jamison, J, D Karlan \& L Schechter 2008, 'To deceive or not to deceive: The effect of deception on behavior in future laboratory experiments', Journal of Economic Behavior \& Organization, vol. 68, no. 3-4, pp. 477-488.

Maniadis, Z, F Tufano \& JA List 2014, 'One Swallow Doesn't Make a Summer: New Evidence on Anchoring Effects', American Economic Review, vol. 104, no. 1, p. 277-290. McDaniel, T \& C Starmer 1998, 'Experimental economics and deception: A comment', Journal of Economic Psychology, vol. 19, no. 3, pp. 403-409.

Milgram, S 1963. 'Behavioral study of obedience', Journal of Abnormal and Social Psychology, vol. 67, no. 4, pp. 371-378.

Mäki, U 2005, 'Models are experiments, experiments are models', Journal of Economic Methodology, vol. 12, no. 2, pp. 303-315.

Ortmann, A 2002, 'The Costs of Deception', Experimental Economics, vol. 131, pp. 111-131.

Schneider, F \& W Pommerehne 1983, 'Free Riding and Collective Action Public Microeconomics', Quarterly Journal of Economics, vol. 96, no. 4, pp. 689-704.

Schram, A 2005, 'Artificiality: The tension between internal and external validity in economic experiments', Journal of Economic Methodology, vol. 12, no. 2, pp. 225-237.

Zizzo, DJ 2010, 'Experimenter demand effects in economic experiments', Experimental Economics, vol. 13, no. 1, pp. 75-98.

\section{Notes}

1. We would like to thank an anonymous referee for helpful comments.

2. Source: http://www.nature.com/news/nobel-laureate-challengespsychologists-to-clean-up-their-act-1.11535 accessed on 2014/5/19. 